\title{
Mathematical Concepts: Fruitfulness and Naturalness ${ }^{1}$ Jamie Tappenden
}

[Speaking of a hypothetical mineralogist who rejects color as a basis for classification, preferring crystalline structure or chemical composition:] The introduction of such a concept as a motif for the arrangement of the system is, as it were, a hypothesis which one puts to the inner nature of the science; only in further development does the science answer; the greater or lesser effectiveness of a concept determines its worth or worthlessness. (Dedekind [1854] p.429)

\section{Introduction}

The preceding paper urged the "Port Royal Principle": "nothing is more important in science than classifying and defining well... [though] it depends much more on our knowledge of the subject matter being discussed than on the rules of logic." This paper will present, in broad outline, a position on mathematical "classification and definition" that is true to the principle and that is interesting both as history and as independently defensible philosophy. An orienting reference is a seemingly innocent passage from an 1899 textbook on elliptic functions:

...the peculiarities of Riemann's treatment lie first in the abundant use of geometrical presentations, which bring out in a flexible way the essential properties of the elliptic functions and at the same time immediately throw light on the fundamental values and the true relations of the functions and integrals which are also particularly important for applications. Second, in the synthetic treatment of analytic problems which builds up the expression for the functions and integrals solely on the basis of their characteristic properties and nearly without computing from the

\footnotetext{
${ }^{1}$ I'm grateful to Paolo Mancosu for comments and patience. Some of this material, in an early form, was discussed in a talk at the Kansas State University philosophy department, and I'm grateful to the participants for helpful discussion. The influence of Hilary Putnam's paper "What is Mathematical Truth?" (Putnam (1975)) runs throughout the discussion of evidence for conjectures. Some of this material was presented to a conference in Princeton in honor of Paul Benacerraf; I'm grateful to the participants - especially Paul Benacerraf, Steven Wagner, John Collins, John Balfe and Hilary Putnam - for comments.
} 
given element and thereby guarantees a multifaceted view into the nature of the problem and the variety of its solutions. (Stahl [1899] p.III italics mine)

We expect to encounter such seemingly generic motivational writing in the prefaces of textbooks, and we tend to flip past to get to the meat. But these words are worth pausing over: through the topic (Riemann's lectures), the means of expression (especially the phrases in italics, which were recognized, red-letter clichés), and the orientation toward "essential properties" gained through the "synthetic presentation" the author displays his allegiance to a methodology and tacit metaphysics of concepts that had been percolating and crystalizing for fifty years, beginning with the revolutionary techniques of Riemann, and winding through the work of his followers (notably Dedekind). ${ }^{2}$ These principles of method were widely enough known that Hilbert needed no further explication for every mathematician to know what he meant when he said that in his approach to higher reciprocity laws: "I have tried to avoid Kummer's elaborate computational machinery so that here too Riemann's principle may be realized and the proofs driven not by calculations but by thought alone." (Hilbert [1897/1998] p.X)

Though the occasional methodological asides are helpful as benchmarks, it is really in the mathematics itself that the power and scope of the revolution in method is manifest. The core idea was that the decision about what to regard as a fundamental property should be seen as part of the problem to be solved rather than as antecedentently evident. (For example, it might be held as evident that + and $\times$ are fundamental concepts and others should be reduced to them. (This was basically Weierstrass' view.) By contrast on the revolutionary view it is possible, and indeed in many cases was concluded, that other concepts should be taken to be basic and representations in terms of + and $\times$ treated as relatively accidental.) The evidence that the selection of basic categories was correct was grounded in the fruitfulness of the relevant formulations for subsequent research. The theoretical outlook and the resulting mathematics have had a profound effect on the emergence of the styles of mathematical reasoning that evolved in the subsequent century.

\footnotetext{
${ }^{2}$ Riemann's basic point of view was interpreted quite differently by different followers. Inspired by Riemann, Felix Klein/Sophus Lie, Alfred Clebsch/Ludwig Brill/Max Noether and Dedekind initiated distinct traditions of mathematics, with different implicit conceptions of method. I believe that the features of Riemann's understanding that I'm describing here are common coin among Riemann's followers, but I will restrict attention here to Riemann as Dedekind understood him, postponing some scholarly subtleties for another time.
} 
My goal here is to describe the Riemann - Dedekind approach to "essential characteristic properties" and indicate some of the mathematics that gives it substance. Along the way, I'll spell out why I regard this as a promising and philosophically profound strategy for arriving at an account of naturalness in mathematical classification that can coalesce with an account of properties and definitions in general. To set the stage, I'll first discuss the current situation in the general metaphysics of properties as it pertains to the naturalness of mathematical properties. The point of the context-setting will be to explain why the way things currently stand - especially the role of metaphysical intuitions, and the stock of examples used as reference points (plus the potential examples that are not used) - make it difficult to address mathematical properties in an illuminating way.

\section{Analytic Metaphysics: The "Rules of the Game" and the Method of Intuitions}

Even if we acknowledge the Port Royal principle of the first essay, and ensure that our account of mathematical "classification and definition" pays due heed to "the subject matter being discussed", we should work toward a synoptic treatment of mathematical and non-mathematical cases. It's unlikely that mathematical and non-mathematical reasoning are so disjoint as to exclude interesting points of overlap. In recent decades there has been a revival of old-fashioned metaphysical debates about the reality of universals, the artificial/natural distinction, and cognate topics. It might seem initially promising to draw on these debates to illuminate the questions appearing in the survey essay. In this section I'll illustrate why the methods accepted as defining the "rules of the game" in the relevant areas of contemporary analytic metaphysics are unlikely to help us as things currently stand. This will force a different perspective on the problem; I'll explore one possibility that centers on inductive practices of conjecture and verification in the subsequent section.

Consider again the example from the survey essay: In algebraic number theory, the definition " $a \neq 1$ is prime if, whenever $a \mid b c$ then $a \mid b$ or $a \mid c$ " is, in an important way, the "correct", or "proper" definition of "prime number", and the school definition " $n \neq 1$ is prime if it is evenly divided by only 1 and n." is comparatively accidental. As in the suggested comparison - the change in the definition of kinetic energy made necessary by relativity theory 
- we need to explain what kind of advance in knowledge it is to replace one definition with another for reasons of the relevant kind. Similarly, we need to clarify what we learn when we arrive at a proper choice of basic categories. Two hundred years of research into number theory reveal that the Legendre symbol determines a central number-theoretic function, carving the subject at its joints, despite the disjunctiveness of the definition that introduces it. But just what it it we are learning when we learn this?

Implicit in the suggestion that finding the right definition or arriving at the proper choice of basic categories can be an advance in knowledge, is the rider that the choice of definition (or of primitive concepts to be defined) can be in some sense "objectively" correct. We are really learning something about the subject we are studying - not just something about ourselves and our cognitive peculiarities - when we learn the "best" definition of "prime", or when we learn that the concept of scheme forms the basis of "the language of algebraic geometry". ${ }^{3}$ What we are learning may be difficult to spell out with precision, of course. To spell it out we may need in addition to clarify some of the other ideas this volume is devoted to (such as mathematical understanding or explanation or purity of method). But the suggestion is that there is some tenable idea of objective correctness to be spelled out.

Of course, this sets a stumbling block: to clarify the suggestion of the last paragraph, we need to flesh out what we mean by "objective". And to be sure, both in the present narrow context and in general, this is not easy to do. Much of the discussion of the objectivity of classifications seems to draw more on some unanalyzed intuitive idea of what "objectivity" must involve, and less on a clearly worked out doctrine or principled analysis. I am in no position to give anything like a principled, general account of a useful concept of "objectivity" here, so my goal will be more modest: to come up with some tangible sufficient conditions for judging a classification to be "objectively correct" in a way that will allow mathematical and non-mathematical cases to be treated uniformly.

For orientation, we'll need to look to the ways that the intuitive idea is unpacked in general metaphysics. The work of David Lewis is useful at this point because it helps bring out what is at stake. Lewis famously adopted a striking change in direction in the early 1980's when he argued that we need

\footnotetext{
${ }^{3}$ Here I am echoing a common way of speaking of schemes, as incorporated for example in the title of the Eisenbud and Harris monograph Schemes: The Language of Modern Algebraic Geometry
} 
to accept a class of properties distinguished as universals. ${ }^{4}$ Lewis' mode of argument is characteristic of his style: he points to the amount of work that the recognition of distinguished properties does, which would be threatened if they were to be given up. This work includes the entries in this list (of course, many of these are close relatives):

1. Underwriting the intuitive natural/artificial distinction in clear cases.

2. Founding judgements of simplicity and similarity (which in turn informs the selection of referents of variables for Ramsey sentences.)

3. Evaluations of general truths as lawlike or not.

4. Supporting assignments of content (for example in "Kripkenstein" cases").

5. Underwriting a distinction between intrinsic and non-intrinsic properties.

6. Singling out "intended" interpretations in cases of underdeterminacy (for example in connection with Löwenheim - Skolem indeterminacy arguments).

7. Distinguishing correct from incorrect inductive predictions in "grue" type examples.

As noted in the introductory essay, there don't appear to be significant differences in the listed respects between selecting distinguished properties in mathematics and in areas dealing with contingent properties of contingently existing things. Two of the cases Lewis treats (Löwenheim - Skolem indeterminacy and "Kripkenstein") directly address mathematical examples. The "Kripkenstein" problem is, in a simple form, the problem of explaining how we come to mean the regular + function when we say "plus", since the specific computations a speaker has performed in a lifetime will always also be compatible with infinitely many other bizarre "quus" functions from pairs of numbers to numbers. Lewis' suggestion is that an answer can begin with the simple observation that interpretations tend to pick out natural candidates rather than wacky ones. The case of inductive prediction might appear to indicate a disanalogy, but as I noted in the introduction, this isn't so. We'll revisit induction in more detail later in this paper. Causation, of course, is a potential spoiler, but it's complicated for reasons indicated in the first essay, and so we'll set it aside here.

\footnotetext{
${ }^{4}$ See Lewis [1983] and [1984].
} 
It is worth making a remark about terminology, to avoid the appearance of prejudging any issues, since "natural" has an unfortunate dual connotation. A choice of categories can be "natural" if it possesses a certain kind of appropriateness or correctness, (as when one says " $\phi$ is the natural map", or "this interpretation is the natural way to understand Kant's remarks on page 17 "), or if it pertains to the physical world (as when one speaks of reducing talk of mental properties to talk of natural properties). Discussion of "natural properties" in metaphysics seem to me most, well, naturally understood as drawing on the former meaning in the cases that are relevant here (such as when "plus" is counted as more natural than "quus", or "grue" less natural than "green") and so it would be natural to speak of "natural properties" in mathematics as well. But it might be objected that speaking of "natural" mathematical functions, definitions, domains, proofs, generalizations, etc. gains rhetorical effect misleadingly from the "pertaining to nature" meanings. ${ }^{5}$ This is a fair complaint, but here I'll have to simply note it as something to keep in mind. It would be difficult to adequately introduce new terminology to disambiguate until we have a clearer sense of what we want the terminology to do. So at this early stage, I'll stick with the common use of the word "natural" and its cognates - ambiguities and all - while keeping alert to the potential for fallacies of equivocation.

If course, part of what gives force to the Lewis treatment is the sheer intuitive umph of some simple common-sense cases. It is hard to deny the prima facie pull of the suggestion that however we understand "objective" it is an objective fact that two electrons $\mathrm{A}$ and $\mathrm{B}$ are more like one another than either of them is like the moon, or the Eiffel Tower, or a moose. This gives urgency to the quest to find an account to either underwrite this intuitive judgement or explain it away. Something like this immediate intuitive force attends the suggestion that "plus" is somehow objectively natural or simple in a way that "quus" isn't. However, such an appeal to the intuitive force of a judgement can hardly suffice for more than preliminary orientation. This is true not just because it is inadequate, in general, to rely solely on brute intuition in philosophical argument, but because the force of some of the better mathematical examples of prospective natural categories ( like "genus" or "scheme") and definitions ("prime") requires training to appreciate. If we can make sense of the idea that categories can be "objectively mathematically natural" in a way that relates to ongoing mathematical investigation

\footnotetext{
${ }^{5}$ I'm grateful to conversations with David Hilbert (the Chicago philosopher, not the Göttingen mathematician) and Hartry Field for helping me see that the ambiguities in the word "natural" could become a distraction in this context.
} 
we'll need to make room for the fact that we can discover that a category is in fact natural even if it seemed to lack intuitive naturalness at the outset. Indeed, in some cases (like the Legendre symbol) the prima facie impression may be that the definition is an obvious disjunctive gerrymander.

Some of the presuppositions about mathematical activity that appear to frame the discussion in the general metaphysics literature present a systematic obstacle to incorporating the relevant mathematical details. It is difficult to arrive at a compelling diagnosis with just vague handwaving about nameless authors. For illustration, I'll look at one representative treatment by Ted Sider [1996] in which mathematical examples are taken to support metaphysical conclusions about the naturalness of properties. Of course, restricting attention to one article gains concreteness but has the potential to sacrifice scope. However, it seems fair to take Sider's paper as a paradigm. Sider is recognized as a significant researcher in the field, the paper appears in a major journal and is widely cited, and my impression is that the intuitions it appeals to are on the whole regarded as acceptable moves by contributors to these debates. I'll leave much of the intricacy of Sider's careful article unmentioned since my concern here is just to use certain aspects of his arguments to illustrate ways in which the presuppositions and accepted moves of current analytic metaphysics make it difficult to address mathematical judgements informatively. In particular, I want to bring out that a general problem with the method of appealing to intuitions about the objectivity of judgements and theories - the extreme context-sensitivity of these intuitions - is especially acute here because of the isolation of metaphysical speculation about mathematics from ongoing mathematics.

Sider sets out to refine an argument of Armstrong ([1986], [1989]) and Forrest [1986] against Lewis. The resulting objection speaks less to the idea of naturalness and more to a further position (called "class nominalism") Lewis endorsed: properties, functions and relations are constructed out of sets. I have reservations about class nominalism, but for orientation it will be useful to accept it and follow out Sider's refinements. ${ }^{6}$

The basic Armstrong - Forrest argument runs as follows: Begin (for the sake of reductio ad absurdum) with the assumption that some relations are

\footnotetext{
${ }^{6}$ Sider suggests that he is not addressing positions that take functions and ordered pairs to be unreduced, sui generis entities. However, Sider's argument against the variation suggested by Phillip Bricker appeals to a premise sufficiently broad as to apply also to any view that takes (unreduced) functions to be distinguished at least in part because of their centrality for mathematical practice.
} 
natural, and choose one natural relation $\mathrm{R}$. The class nominalist reduces relations to sets of ordered pairs, and ordered pairs to sets. There are different ways to do this. The reductions considered explicitly are Kuratowski's definition $\langle x, y\rangle=\{x,\{x, y\}\}$ and Weiner's $<x, y>=\{\{x, \emptyset\},\{y\}\}$. If $\mathrm{R}$ is natural, then the collections of sets corresponding to $\mathrm{R}$ should be natural. The objection is: It is arbitrary which reduction of ordered pairs is adopted, which conflicts with the sought - after objective, non-arbitrary character of the assignment of naturalness to the set that represents $R$. The point, as so distilled, needs fleshing out and strengthening, but the core idea is clear enough. Sider's paper suggests a sequence of such fleshings out and strengthenings, followed by his criticisms of these proposals, before arriving at what he takes to be tenable final results.

Sider considers this reply to Armstrong - Forrest: perhaps one of the reductions should be counted as the right one. Sider suggests that there couldn't be a reason for preferring one over the other, which seems plausible in the specific case of these reductions of ordered pairs. It's less clear what to say about another example Sider considers: the well-known "multiple reduction problem" first broached in Benacerraf's "What Numbers Could not Be" [1965]. It is observed that the natural numbers can be reduced to either of two series: Zermelo's $\{\emptyset,\{\emptyset\},\{\{\emptyset\}\}, \ldots\}$ where each member of the series is the unit set of the previous one, or Von Neumann's $\{\emptyset,\{\emptyset\},\{\emptyset,\{\emptyset\}\}, \ldots\}$ where each member of the series is the set of its predecessors. The suggestion that one reduction or the other might be correct is rejected on the grounds that a reason to prefer one over the other is inconceivable: "Perhaps one line of our thought here is that we don't see what could possibly count as a reason" ([1996] p.289) and "The only features that distinguish one method from another involve mathematical convenience, and so seem irrelevant to the existence of an ontologically distinguished method." ([1996] p.289)

Sider addresses another variation on the Armstrong-Forrest point. Say that instead of arguing for a single distinguished method, we accept that there will be many, and maintain that all of them are equally natural. Sider rejects this on the (reasonable) ground that some methods are obviously unnatural, and he produces a grotesquely gerrymandered case to witness the point. Thus we come to what seems to me the best option (given an antecedent acceptance of class nominalism): Say that there is a class $\mathrm{C}$ containing more than one method, such that no method is more natural than any member of $\mathrm{C}$, every member of $\mathrm{C}$ is equally natural, and every method as natural as a member of $\mathrm{C}$ is in $\mathrm{C}$. Say also that there is at least one method that is less natural than the members of $\mathrm{C}$. That is, $\mathrm{C}$ is the class of methods 
such that nothing is any better than a member of $\mathrm{C}$. Why shouldn't we just pick an arbitrary member of $\mathrm{C}$ and stick with it, so long as we avoid the less natural methods not in $\mathrm{C} ?^{7}$ The fact that there are several winners in equal first place doesn't take away our ability to distinguish more and less natural. ${ }^{8}$

Sider's only grounds to reject this option is to amplify the claim noted above: "While a class of distinguished methods may be less implausible than the single distinguished method... it is still implausible. [This] theory seems to mistake a pragmatic distinction (Kuratowski's method is more mathematically convenient than method X) for an ontological one. Thus, I continue to reject the idea that any method of constructing ordered pairs is ontologically more distinguished from any other." (1996 p.292) Given that the "method X"'s at issue include the obviously gerrymandered case whose intuitive unnaturalness Sider appealed to, and indeed any reduction however artificial and clumsy, Sider's claim will only do the work he needs if it is understood broadly enough that any distinction between natural and unnatural mathematical reductions, if made on the basis of an assessment of mathematical naturalness, is counted as based on merely "mathematical convenience", which is to be counted as merely "pragmatic" and not an objective guide to the way things are.

Two related suggestions underwrite Sider's stance: the purported inconceivability of any reason to prefer one reduction (of numbers, and by implication of ordered pairs), and the claim that any reason that could be adduced could only be a matter of "mathematical convenience", and consequently a "pragmatic" distinction rather than an "objective" / "ontological" one. Many theoretical choices made in the course of successful mathematical reasoning would be thereby shrugged onto the "merely pragmatic" scrapheap, so we should pause and take stock. The plausibility of these claims rests significantly on the examples taken as paradigmatic. If we restrict ourselves to ordered pairs, it does indeed seem inconceivable that there could be a substantial, non-pragmatic reason to prefer Kuratowski over Weiner. But it is a

\footnotetext{
${ }^{7}$ The situation would be like the one we face when choosing a coordinate scheme for Euclidean space. Of course, there is no uniquely reasonable choice. Does this mean that every choice is equally good? Of course not: some assignments of algebraic objects to geometric ones are terrible. Say, for instance that we have Cartesian coordinates and polar coordinates in alternating octants. Then the mathematical description of (say) a sphere centered at the origin would be wild indeed despite the mathematical simplicity of the figure described, and that would be the fault of the choice of unnatural coordinates. Here it seems right to say that there are a class of distinguished coordinatizations, all more or less equally acceptable, and all superior to a range of unacceptable ones.

${ }^{8}$ Sider attributes this alternative to Phillip Bricker.
} 
mistake to generalize this impression, because of the mathematical insignificance of the example. If "mathematical convenience" means "convenience for the practice of mathematics" then the reduction of ordered pairs to sets is at best valueless. In mathematical practice, reduction for the sole purpose of paring down the number of basic entities is regarded with indifference or even distaste. (Reductions can be valued, of course, but only if they bring some kind of mathematical benefit, as measured by, for example, improved understanding or enhanced potential for discovering proofs, or "purity of method" considerations of the sort considered in Mic Detlefsen's contribution.) The reduction of ordered pairs to sets has been used by analytic metaphysicians as a paradigm of mathematics since Quine singled it out as one of his canonical examples. But it is not a paradigm of mathematics. In fact, the reduction of ordered pairs to sets has become a stock example in philosophical discussions because it fits with widely shared and well-entrenched philosophical assumptions. Quine took it as a paradigm of ontological economy, and ontological economy was proposed as a core methodological objective. Through an unnoticed philosophical metamorphosis, ontological economy came to be counted as a measure of simplicity, with simplicity of the relevant kind counted as a virtue of scientific theories.

Of course, even those who hold that some preferences for one representation over another are based on reasons of more than "mere convenience" will grant that some such preferences are just based on relatively trivial pragmatic grounds. Recall that, as noted in the introductory essay, we can cite several reasons for not counting 1 as a prime number. Some of these involve deep facts about the behavior of units in general fields, others can reasonably be seen as minor improvements in streamlined bookkeeping, such as the availability of a compact statement of the prime decomposition theorem. It's plausible that any reason for preferring the Weiner reduction over Kuratowski or conversely could only cite bookkeeping advantages. But the tacit suggestion that this comparison is paradigmatic of mathematical choices of this type masks just what a range of distinct, subtle considerations will get shrugged onto the "pragmatic" heap.

The point comes out more pressingly in connection with Sider's example of the natural numbers. Though the accepted philosophical folklore holds otherwise, there are mathematically important differences between the Von Neumann and Zermelo representations. Thanks to these differences, Von Neumann's series has long been accepted by set theorists as the most natural, canonical representation of $\mathbb{N}$ and Zermelo's is a forgotten historical curiosity. It is important to recognize that substantive reasons can be cited 
for this; it is not just a contingent historical accident. (The Von Neumann ordinals are not merely VHS to Zermelo's Betamax.) It would be distracting to dissect all the reasons (some of them are rather involved), so I'll concentrate on just a particularly clear and simple one: The principle generating the finite Von Neumann ordinals generalizes naturally to infinite numbers while the one generating Zermelo's ordinals doesn't. Take $\omega$, the ordinal of the series $\{0,1,2,3, \ldots\}$. A von Neumann ordinal is the set of its von Neumann predecessors, so the principle generating the finite ordinals gives us immediately the representation $\omega=\{\emptyset,\{\emptyset\},\{\emptyset,\{\emptyset\}\}, \ldots\}$ A Zermelo ordinal is the unit set containing its predecessor, which for $\omega$ is... well, $\omega$ has no predecessor. So there is no Zermelo $\omega$.

We'd need to jigger up something else to regard the Zermelo ordinals as the finite initial segment of all the ordinals. Of course, isn't too hard to come up with something. For example, we could make every successor ordinal the unit set of its predecessor, and every limit ordinal the set of all its predecessors. But this would evidently be an artificial patch job, and a uniform account would be preferable. Though the von Neumann/Zermelo hybrid might occasionally be clumsy to work with, the problem with it is not inconvenience but rather unnaturalness. A proper account of the ordinals should display finite and infinite ordinal numbers as the same kind of thing, with ordinals in general as a natural generalization of the finite ordinals. Whether or not finite and infinite numbers should be generated by the same or different principles is the sort of thing that should be regarded as a fundamental question, contributing to an at least potentially objective case for one representation over the other.

This serves up a striking example of the context-sensitivity of the metaphysical intuitions at work in this debate. If the debate is carried out in isolation from the ongoing mathematical research that is ostensibly its subject, we should expect situations like this to arise: The non-existence, and indeed the inconceivability of a kind of mathematical argument is put forward as a central datum, when such arguments are not only possible but are widely, if tacitly, acknowledged in practice. In addition, with the reduction of ordered pairs taken as a mathematical paradigm, the student of mathematical method is put in a difficult dialectical position: the reduction that is put forward as fulfilling mathematical desiderata does indeed seem arbitrary and artificial. It is indeed hard to see how either reduction of ordered pairs could contribute to our view of what reality really is like. But from the point of view of mathematical method, it is appropriate to think this: the reductions are mathematically artificial, whatever their philosophical virtues may be. 
One reason that informal mathematical reasoning of this type has stayed off of philosophy's radar is hinted at in a remark Sider quotes as sympathetic to his position:

In awaiting enlightenment on the true identity of 3 we are not awaiting a proof of some deep theorem. Having gotten as far as we have without settling the identity of 3 , we can go no farther. We do not know what a proof of that could look like. The notion of "correct account" is breaking loose from its moorings if we admit of the possible existence of unjustifiable but correct answers to questions such as this. (Benacerraf (1965) p. 58)

Certainly proving theorems is the canonical means of obtaining knowledge of mathematical facts. If we narrow our picture of the cognitive activity informing mathematical reasoning to include just deductively valid arguments from indubitable premises, then we won't have any access to the sorts of reasons that we are interested in here. The arguments that can be given to justify counting one definition as "correct" or one reduction as "natural" are not going to be theorems. (Of course, sometimes central theorems can be crucial to making a case for the naturalness of one definition or function over another. This was the case for the Legendre symbol: its claim to the status of natural rather than artificial was buttressed when the Artin Reciprocity Theorem was proven, and it turned out to be the special case of a more general fundamental concept.) But the fact that this reasoning is "softer" than what we are accustomed to finding in mathematics textbooks doesn't mean it is an insignificant contributor to mathematical knowledge. The principles provide reasons for mathematical expectations, guide conjectures and inform problem-solving strategies. The reasons can be debated, and those debates can be resolved by further investigation. Why shouldn't we count this as rational activity producing objective knowledge?

At its crucial points, Sider's argument seems to amount to simply appealing to the perceived plausibility of the suggestion that mathematical judgements of more or less natural can never be more than judgements of "mathematical convenience" that cannot be reasonably taken as a guide to the way things are objectively. Part of what gives initial appeal to this claim is the example of the reduction of ordered pairs, which I've suggested is misleading as a guide to the judgements informing mathematical practice. A 
mathematically insignificant case is taken as paradigmatic, which colors the intuitions about the other cases it is meant to illustrate. Of course, this observation puts the ball in my court: I'll need to lay out other examples which will turn the intuitions around. The examples developed in the first essay can take some steps in the right direction. Given the extensiveness, intricacy, and multiplicity of connections exhibited by the reasoning at issue it doesn't seem nearly so obvious that we can devalue as a judgement of "mere mathematical convenience" the claim that the proof of quadratic reciprocity using cyclotomic extensions is especially natural because it provides a conceptual stepping stone to more general Artin reciprocity, or that the Legendre symbol, despite the artificial-seeming initial definition, can be seen to carve things at the joints when it is recognized as a special case of the Artin symbol.

Of course, the topic of context-sensitivity is a double-edged sword here. It is open to the defender of the thesis that judgements of mathematical naturalness are all ("merely") pragmatic to suggest that familiarity with mathematical details distorts intuitions as well. Immersion in the details of any rich theory with a range of systematic interconnections can foster a sense that certain facts that may seem soft from the outside are hard and inescapable. This is true for systematic theology, chess theory, and the theory of what Harry Potter would do under hypothetical circumstances consistent with, but not occurring in, the Harry Potter books. Enough immersion in the Potter books, discussion groups with other fans, fan fiction groups on the internet etc. might give a fan the sense that it is an objective fact that one continuation of the story is natural and another is artificial, and the fan might be able to provide an extensive and ingenious rationale. A well developed theory of the Potter stories could provide explanations of the actions of the characters, and could be assessed for simplicity, elegance, and Potter theoretic analogues of "purity of method" considerations, to mention just a few cognitive virtues. But a skeptic could maintain, quite plausibly, that this doesn't make the naturalness of one storyline versus another anything but an artifact of the cognitive peculiarities of the enthusiastic fan, and perhaps of human cognitive particularities generally. Not unreasonably, the defender of the thesis that judgements of mathematical naturalness are all pragmatic could point to such analogies, to argue that it is just irrelevant that Sider considers a trivial example instead of richer cases with complex details.

At bottom, this reply could run, the preference for one mathematical formulation over another, in principle logically equivalent one, is a pragmatic matter, however detailed the rationale, however intricately the preferences may be bound up with attributions of mathematical understanding or ex- 
planation or simplicity or [insert your favorite virtue of theories here], and however subtlely the preferences may interact with problem - solving potential. The mere fact that the judgements are deeply embedded in the practice of mathematics does not change their nature. It may make the pragmatic judgements more complicated, and they may "feel" differently to people familiar with the subject, but they remain ultimately pragmatic, just as artistic judgements about musical works are not changed into something other than aesthetic judgements if they are complex and involved.

However, as other strands of the Sider article bring out, the contextsensitivity of intuitions about objectivity is a universal concern. Following out a different cluster of options, Sider takes up the possibility that the idea of naturalness might admit of many subgenres, depending on what reduction is accepted. (So, there could be "Kuratowski - natural" relations, "Weiner - natural" relations, and so on, but no generally definable class of natural relations.) The sole grounds given for rejecting this option are appeals to intuitions about complexity and the aesthetics of theories: "[This is not] a knockdown objection, but [it is] a forceful one nonetheless: [The theory] would require an infinitude of primitive properties, with no hope of subsumption under a single formula or explanation. If [the theory] requires such an unlovely menagerie to do its work, we'd do better to look elsewhere for a theory of naturalness." (p.293) This objection is redescribed later as an appeal to "theoretical economy" (1986 p.295) and later still as an appeal to "prohibitive cost in complication of theory" (1986 p.299). The obvious question presents itself: why are offhand intuitive assessments of simplicity and aesthetic appeal reliable guides to the way things really are for philosophical theories but pragmatic matters of "mere convenience" for mathematical discriminations? It is hard to see any principled basis motivating the distinction in the cases at hand.

This draws us to a deep problem to be sure, but it is a problem for everyone: why should theoretical virtues serve as a guide to the way things are? How can we justify an appeal to relative simplicity of one account over another, or the fact that one account explains or confers understanding better than another, or some other cognitive advantage, when there would seem to be no a priori reason to expect that objective reality must be so considerate as to conform to the particularities of human cognitive makeup. There is no generally accepted response to the suggestion that every choice of a theory in mathematics, philosophy, or any other field - on the grounds of theoretical virtues like simplicity or apparent naturalness or "loveliness" is "merely pragmatic". So for any particular, local appeal to such theoretical reasons, 
the charge that these reasons are "merely pragmatic" is available. If we want to maintain that even so, some apparently pragmatic judgements are guides to the way things objectively are and others are not, we need a solid basis for distinguishing the cases. The problem with the method of appealing to intuitions about what is and isn't "pragmatic" is that these intuitions are simply too context-sensitive to afford an informative distinction between cases where a preference motivated by theoretical virtues is "objective" and when it isn't.

In the end, it is hard to make any progress beyond a wheel-spinning clash of intuitions without more information about what "objective" means, or some harder criteria of objectivity. But if our intuitions are too fluid to adjudicate whether or not some discrimination is objective, what else is there?

\section{Conjecture and Verification: a Foothold on Objectivity}

As I've said, I am in no position to propose a general theory of, or criterion for, the objectivity of a judgement. Rather, I'll borrow a practical strategy from mathematical research: if you can't solve the general problem, find a tractable special case and solve that. The foothold will be the interaction between finding the proper definitions or concepts and the practice of successfully verifying conjectures. This won't be the whole story but it can be the beginning of one. In particular, I'll concentrate on one orienting mark: it is a prima facie sign that a judgement is objective if it has consequences that can be confirmed or refuted and whose truth or falsehood is independent of the original conjecture. In the simple cases that we know from discussions of the "grue" paradox, these consequences are successful empirical predictions. As noted in the introductory essay, such predictions are made in mathematics as well, as witness the inductive reasoning Euler exploited to arrive at quadratic reciprocity. Euler's argument for quadratic reciprocity forms a pleasingly clear-cut analogy to Goodman's New Riddle of Induction. In each case we are dealing with simple enumerative induction, and the correctness of the prediction depends upon hitting on the proper category. The fact that one is a prediction about a contingent fact and the other a necessary truth about prime numbers makes no difference to the reasoning itself.

One of the reasons that the Goodman's "New Riddle" is such a compelling philosophical set-piece is that it links - in a simple and clear way - the ques- 
tion of whether or not a category is homogeneous with a judgement whose rightness or wrongness is outside the control of the judger. No amount of reclassifying or pragmatic revision of conceptual schemes will make the next emerald examined be (what is called in the current vocabulary) blue. The color of an emerald is a contingent empirical fact, but as noted the reasoning doesn't turn on contingency. Euler's records of the computations that prompted his conjecture of the law of quadratic reciprocity display the same pattern. ${ }^{9}$ He examined cases, made conjectures and then tested them against an expanding series of examples. As with induction about the physical world, Euler's analyses of the data were, of course, not unreflectively mechanical. It took considerable ingenuity and pattern - spotting before Euler recognized that (what amounted to) quadratic reciprocity was the pattern to project. After he hit on the proper way to classify the cases he had examined, he was able to predict the correct theorem, which was then verified through examination of further specific values. It is remarkable how well the patterns of computation, and conjecture, refute conjecture with more computations, refine concepts and make new conjecture,... look like textbook examples of enumerative induction. (Find some green rocks. Conjecture all rocks are green. Find some non-green rocks, re-analyze situation, note that the green rocks are emeralds. Conjecture all emeralds are green ...) To the extent that the naturalness of a property plays a role in the correctness of a projection, it has to play a role here, which would lead us to the conclusion that the properties like " $\mathrm{x}$ is a quadratic residue" supporting Euler's correct inductive reasoning should have the same claim to "naturalness" deriving from projectibility that "green" has. The elementary inductive pattern is in no way altered by the fact that the theorem could also be directly proven, or that the relevant facts about numbers aren't contingent, and the pattern is perfectly consistent with the observation that the concept of causation doesn't have any obvious application in connection to mathematical properties. The correctness or otherwise of the categories Euler used was tested by a hard, objective criterion: will the values still to be computed be the predicted ones? And further down the road: if the conjecture is resolved by a proof, will it be proven true or false?

Of course, the Euler case is unusual, in that elementary enumerative induction of the form " $a_{1}$ is $P, a_{2}$ is, $P, \ldots, a_{n}$ is $P$, therefore $a_{n+1}$ will be $P$ " is as rare in mathematics as it is in ordinary reasoning about the physical world:

\footnotetext{
${ }^{9}$ There are several excellent treatments of the inductive reasoning that led Euler to his conjecture. Edwards (1983), Cox (1988) and (1989 p. 9 - 20), and Weil (1984) are all worth consulting. Sandifer (2005) is a particularly beginner-friendly discussion at the online Euler archive.
} 
both ordinary and mathematical conjectures are typically more subtle. ${ }^{10}$ In both empirical and mathematical cases, the simple enumerative induction is an artificially simplified touchstone that distills certain essential features of more formless kinds of prediction and conjecture that inform ongoing mathematical and empirical thinking.

The practice of conjecturing and then striving to ascertain whether or not the conjecture is correct is ubiquitous in mathematical practice. ${ }^{11}$ Even without the formality of explicitly announcing a conjecture, the practice of forming expectations about what will turn out to be correct, grounded in prior discoveries, is an indispensible component of mathematical reasoning. These expectations guide the choice of directions to search for proofs, for example. The concepts that are marked out as mathematically natural support further inductive practice: on the basis of similarities that are evaluated using these concepts, conjectures are made about what further investigations will discover. If it is discoved that these conjectures are true, it reinforces the judgment that the concepts regarded as mathematically natural really are mathematically natural. In at least some cases the expectations are sufficiently well-grounded in computations or other quasi-empirical data or plausibility considerations that it seems right to say that the theorems are known to be true even without a proof. (This seems to be a reasonable thing to say about Euler's justified belief in quadratic reciprocity, for example.)

Inductive reasoning doesn't just appear in the direct form of conjectures or specific expectations that are then ideally refuted or verified. Broader methodological virtues like "significance" and "fruitfulness" have a kind of quasi-empirical inductive character. ${ }^{12}$ Singling out a property as central tacitly makes a prediction that it will reappear in unexpected contexts, and serve as support to unanticipated proofs. It need not be obvious at the outset what shape the proofs will take or what the "unexpected contexts" will be for this to be a prediction that can succeed or fail. This supports a re-

\footnotetext{
${ }^{10}$ Though there are significant examples in which an approach is initially validated through its ability to make verifiable predictions. Perhaps the most famous is the Schubert calculus for counting intersections of curves. Long before the system could be rigorously formulated and demonstrated, Schubert was making astonishing predictions of intersection numbers. See Kleiman and Laksov (1972)

${ }^{11} \mathrm{~A}$ good recent discussion of conjecture as an aspect of mathematical reasoning is in Mazur (1997). The classic investigations of inductive reasoning in mathematics are of course Polyá (1968) and Lakatós (1976). Putnam (1975) is an illuminating reflection on the philosophical angles. Corfield ((2003) chapters 2-6) contains some valuable discussion.

12 "Quasi-empirical" is the expression coined by Hilary Putnam for this sort of mathematical evidential support in his "What is Mathematical Truth" (Putnam (1975)
} 
sponse to the suggestion that these evaluations are "merely pragmatic" or "subjective" or "merely psychological" or otherwise lacking objectivity in a way that makes them uninteresting to epistemology. Just as with more narrowly circumscribed conjectures, the predictive character of an evaluation of "fruitfulness" brings a degree of independence from the attitudes of the person making the judgement. To judge that a program, theorem, or definition is "fruitful" is to make a prediction that solutions to given problems will in fact be found by means of the program, theorem or definition. Such judgements involve previsions that can be found to be mistaken in light of subsequent investigation. Not all predictions are as unequivocal and precise as Euler predicting quadratic reciprocity, but they can still be confirmed or disconfirmed. There is, of course, a social component to the prediction that some approach will yeild research deemed to be valuable and informative. But a prediction like "The Weil conjectures will be solved using the concept of scheme and not with the concepts available earlier." is not just a forcast of social facts. It turns both on what mathematicians will choose to do and on what proofs are objectively there to be found.

In stating that inductive and plausible arguments in mathematics can secure knowledge, I am not echoing the suggestion one occasionally encounters that the mathematical community ought to relax its emphasis on rigorous proof. Something can be known without being established to the degree required for the mathematical community to count it as officially in the bank. (Analogously, I would not be suggesting that criminal trial procedure should relax the standard of "proof beyond a reasonable doubt" if I were to point out that we know certain former defendants to be guilty even though they were ruled not guilty at their trial.) I am suggesting no revisions at all to regular practice. I am rather urging that we extend the range of mathematical reasoning that we take to generate knowledge and to be material for epistemological study. Forming expectations on the grounds of plausible reasoning and using these expectations as a guide is an integral part of mathematical practice. My point has been that if we expand our epistemological horizons to include these varieties of mathematical knowledge, it brings with it an additional bonus: A foothold on the study of the naturalness of mathematical concepts and definitions.

I should also make clear that in urging the importance and quasi-empirical character of the tacit predictions informing mathematical reasoning, I am not suggesting that our study of mathematical concepts must be linked to direct applications in physical science and commonsense reasoning about the physical world. (Of course, in some cases there will be close connections, but this 
isn't necessary to the point. ${ }^{13}$ Whether or not the conjectures in question have any obvious connection to any direct applications makes no difference to our assessment of the patterns of reasoning informing the making and supporting of conjectures. It may well be that in an ideal epistemology, the status of mathematics as knowledge must ultimately appeal to physical applications, or it may not; we can be neutral on that point here.

\section{Fruitfulness, Conjecture and Riemannian Mathematics}

The Riemann-Dedekind methodology mentioned in the introduction incorporates exactly the connection between identification of core properties and mathematical fecundity discussed in the previous section. In this section I'll say a bit more about the Riemann-Dedekind approach. First consider these Riemannian remarks. They seem innocuous and even dull, but understood in context they are the first shot in a revolution.

Previous methods of treating [complex] functions always based the definition of the function on an expression that yields its value for each value of the argument. Our study shows that, because of the general nature of a function of a complex variable, a part of the determination through a definition of this kind yields the rest... This essentially simplifies the discussion...

A theory of these functions on the basis provided here would determine the presentation of a function (i.e. its value for every argument) independently of its mode of determination by operations on magnitudes, because one would add to the general concept of a function of a variable complex quantity just the attributes necessary for the determination of the function, and only then would one go over to the different expressions the function is fit for. The common character of a class of functions formed

\footnotetext{
${ }^{13}$ Though I'm not exploring the point here, a closeness to physical applications was in fact recognized as a feature of Riemann's mathematics. (This is touched on in the opening quotation from Stahl.) For example, Helmholtz found Riemann's complex analysis congenial, presumably in part because Riemann's classification of functions by their singularities fit smoothly with Helmholtz's approach to potential flow. (cf. Darrigol [2005] p.164)
} 
in a similar way by operations on quantities, is then represented in the form of boundary conditions and discontinuity conditions imposed on them. (Riemann [1851] p.38)

Riemann's point is simple. We are wondering how to characterize a wellbehaved complex function or a class of them. How should we proceed? One possibility would be to appeal to a formula that would explicitly display the relation between every argument and every value in terms of elementary operations like plus or times. For example, one could write down a polynomial or power series. But this uses more information than necessary: the function can be uniquely determined (up to a constant multiple) by just a fragment of this information (the "boundary conditions and discontinuity conditions").

I'll illustrate with an example where the conditions are particularly straightforward: elliptic functions, the subject of the Stahl textbook quoted in the introduction. $^{14}$ These are (defined today as) well-behaved (i.e. meromorphic) functions $\Phi$ satisfying the condition that there are complex numbers $\omega_{1}$ and $\omega_{2}$ such that for any $z \in \mathbb{C}$ and $n, m \in \mathbb{Z}, \Phi(z)=\Phi\left(z+n \omega_{1}+m \omega_{2}\right)$ (i.e. $\Phi$ is doubly periodic.) Given periods $\omega_{1}$ and $\omega_{2}$, how should we single out specific functions? We could give an explicit rule for computing every value for every argument, but we have a sparer alternative. Fixing the natural domain (period lattice) corresponding to the periods $\omega_{1}$ and $\omega_{2}$, given any two functions $f$ and $g$ on this domain with the same zeros and poles (counted with the same multiplicities) there is a constant $c$ such that for every $z, f(z)=c g(z)$. That is, the function is essentially fixed by its zeros and poles; the remaining values need be pulled up only if necessary for some specific purpose. Though I am restricting attention to a simple case to avoid discussion of Riemann surfaces, the fact holds more generally: we lose no important information about a well-behaved function if we just know the zeros and certain singularities. ${ }^{15}$

With such results in hand, Riemann opts to characterize functions by their singularities. There are too many ramifications of this preference to discuss here. I'll restrict attention to the one that is directly relevent to our present topic: getting the concepts right allows you to see results more

\footnotetext{
${ }^{14}$ Here I'll only be able to gesture at some of the relevant information. I explore elliptic functions in connection with Riemann's conception of mathematical method in more detail in Tappenden [200?].

${ }^{15}$ Given any compact Riemann surface $R$, a meromorphic function defined on $R$ is characterized up to a constant by its zeros and poles (with multiplicities).
} 
easily. ${ }^{16}$ For example, concerning one application of his methods, Riemann deploys the language that gave rise to the clichés echoed by Stahl: Riemann's reconceptualizations make it easy to recognize at a glance what had previously required effort and laborious computations.

[My method yields] almost immediately from the definition results obtained earlier, partly by rather tedious computations. (Riemann [1857] p.67)

[My method is one] by means of which all the results found earlier can be obtained virtually without computation. (Riemann [1857a] p.85)

Not only does the reconceptualization allow you to see easily what had previously been established with difficulty, but also as Dedekind explicitly notes, the temporal order can be reversed. The formulation "based immediately on fundamental characteristics" supports the effective anticipation of the results of as yet unfinished computations. Dedekind sees "almost all areas of mathematics" as requiring the choice between essential ("internal") and accidental ("external"):

It is preferable, as in the modern theory of [complex] functions to seek proofs based immediately on fundamental characteristics, rather than on calculation, and indeed to construct the theory in such a way that it is able to predict the results of calculation ... Such is the goal I shall pursue in the chapters of this memoir that follow. (Dedekind [1877/1996] p. 102)

[Gauss remarks in the Disquisitiones Arithmeticae]: "But neither [Waring nor Wilson] was able to prove the theorem, and Waring confessed that the demonstration was made more difficult by the fact that no notation can be devised to express a prime number. But in our opinion truths of this kind ought to be drawn

\footnotetext{
${ }^{16}$ Among the other considerations Riemann cites are purity of method considerations arising from his sense that it isn't proper for language-independent properties of objects to be characterized in ways that essentially involve language:

By one of the theorems quoted above, this property of single-valuedness in a function is equivalent to that of its developability in a series...However, it seems inappropriate to express properties independent of the mode of representation by criteria based on a particular expression for the function. (Riemann [1857b])
} 
out of notions not out of notations." In these last words lies, if they are taken in the most general sense, the statement of a great scientific thought: the decision for the internal in contrast to the external. This contrast also recurs in mathematics in almost all areas; [For example, complex] function theory, and Riemann's definition of functions through internal characteristic properties, from which the external forms of representation flow with necessity. (Dedekind [1895] p. $54-55$ )

In some cases, such as the zeros and poles of a complex function, the "internal characteristic properties" at issue are already familiar, so the cognitive success involved in identifying them is just the recognition of their importance. In other cases, it requires substantial reformulation and analysis even to be in a position to notice and define the properties. Thus, for example, Riemann recognized the importance of the property we now call the genus of a surface in connection with complex functions. (In the simplest cases, the genus is the "number of holes" in the surface.) Riemann showed how to associate with each well-behaved complex function a surface (now called a Riemann surface) serving as the natural domain of definition for the function. The genus is then defined in terms of the topological properties of this surface. This sets the context for a core result proven partly by Riemann and partly by his student Roch (called, reasonably, the Riemann - Roch theorem) that had incalculable importance in the mathematics of the twentieth century. Here again I can hardly give more than a vague impression, so I'll just state the punchline for this paper: The result links together the number of linearly independent functions with given collections of poles defined on a Riemann surface with the genus of the surface. The subsequent importance of the theorem, and the fact that it links together the properties that would have been understood as the kinds of things being seen as "inner characteristic properties" makes it one of the many examples that give mathematical content and force to Riemann's and Dedekind's methodological asides. The characterization of poles and genus as essential properties is tacitly a prediction that the Riemann-Roch theorem, among others, will prove fruitful in solving additional problems and supporting new conjectures. This is a chancy prediction, since the theorem may have led nowhere. Had the research jumping off from core theorems about genus and poles of functions petered out without interesting consequences, this would have forced a reassessment of the original evaluation of these properties as "internal characteristic" ones.

The most salient example of his own work that Dedekind is alluding to in the above remarks is the concept of an ideal. Once again there is much more 
to discuss than I can address here, so I'll just stick to the immediately relevant punchlines. ${ }^{17}$ It will suffice to think of an ideal as a kind of generalized number that secures the possibility of factoring otherwise irreducible numbers. Among the discoveries that gave impetus to Dedekind's introduction was an analogy between fields of numbers and fields of functions unveiled in the classic paper by Dedekind and Weber [1882]. ${ }^{18}$ (Riemann's characterization of functions by their zeros and poles was a crucial building block.) Among other things, Dedekind and Weber interpreted Riemann surfaces as algebraic objects and proved core results such as the Riemann-Roch theorem in this environment. Speaking informally we might say that Dedekind and Weber revealed that a family of structures were genuinely, rather than just superficially similar. The analogy between fields of numbers and fields of functions is very deep.

It is possible to lay out an extensive rationale for the judgement of similarity. ${ }^{19}$ As just one illustration of how layered the judgement of similarity can be, consider the property of ramification for complex functions and generalized numbers. ${ }^{20}$ Among the singularities Riemann recognized to be especially important are branch points (a.k.a. ramification points). With proper stage setting, these can be understood as points where the function behaves locally like $z \mapsto z^{e}$, with $e>1$. When dealing with number fields, the idea of prime decomposition can be generalized so that generalized numbers (ideals) can be written as products of generalized primes $\mathfrak{P}_{i}$ :

$$
\prod_{i=1}^{n} \mathfrak{P}_{i}^{e_{i}}
$$

The generalized number is said to ramify if $e_{i}>1$ for any $i$. This point of contact between complex analysis and algebraic number theory was already pointed out by Dedekind and Weber.

\footnotetext{
${ }^{17}$ Fortunately for any readers hungry for more, the relevant details are well covered by historians, in particular Harold Edwards. See for example Edwards [1980]. Recently philosophers have begun to reflect on the methodology described in Dedekind's remarks and displayed in his mathematics. See for example Avigad [2006] and my [2005].

${ }^{18}$ This analogy, with specific reference to ramification, is explored with a philosophical eye in Corfield [2003] (p.90-96)

${ }^{19}$ An illustration can be found in a letter of 1940 from André Weil to his sister Simone, containing a strikingly detailed discussion of the many consequences of this analogy, what matters in the analogy, what is needed for the analogy to be complete, etc.. (Weil also spells out connections to quadratic reciprocity and the Artin reciprocity theorem.) See Weil [1940/2005])

${ }^{20} \mathrm{I}$ 'm indebted to Brian Conrad for illuminating conversations on this point.
} 
Of course, in one sense this particular connection is laying on the surface of the algebraic representations. In both cases you have exponents either greater than one or equal to one - sitting right there just waiting to be noticed. But if all we know is that some exponent is greater than one, we have no reason to get excited, or judge there to be a point of similarity robust enough to warrant the introduction of new terminology. The point of similarity could be a superficial accident of the representation, or a mathematically inert coincidence. The bedrock support for the claim that this is a genuine point of deep similarity is the fact that powerful general theorems can be proven which exploit the analogy. Had it turned out that, at a more advanced point of reckoning, no interesting theorems emerged, the shared terminology would stand like the word "elliptic" in the phrase "elliptic curve" as a quaint reminder of a connection that once seemed to matter. As always, the aesthetic judgements, assessments of the depth of a similarity, judgements about appropriateness of techniques, evaluations of relative simplicity, etc. are subordinated to the fundamental bottom - line consideration: does it lead to genuinely interesting new results? Without the sine qua non of fruitfulness, the other considerations are counted as ultimately incidental.

Riemann's envisioned connection between correct definition and fruitfulness was revolutionary at the time, but now seems familiar, even banal. Indeed, it is fair to say that it is the dominant attitude among contemporary mathematicians. It is hardly profound or surprizing that mathematicians typically have a hardheaded, bottom-line orientation that ties the ultimate validity of softer judgements of "naturalness" or "depth" to the facilitation of far-reaching general results. This piece of rudimentary empirical sociology is obvious to anyone after a few department lounge conversations. Of course, for Riemann and Dedekind, and even more for most contemporary mathematicians, the attitude is more often displayed in the practice of mathematical research than articulated as something like a philosophical theory of the natural/artificial distinction. But there is a tacit theory behind the pronouncement that (say) genus is a natural property and as philosophers we'd be wise to work it out.

\section{Summing up}

The introductory essay made a descriptive observation and posed a problem. The descriptive observation is that mathematical practice is colored 
and guided by a kind of normative judgement: it can be counted as an advance in knowledge to identify a "natural" definition (or "deep" analogy, or "proper" context for investigation, or "correct" formulation of a question ...). The problem is to find a way for epistemology to explore how such acts of recognition can be what mathematical practice seems to treat them as being. This paper has been an extended prospectus for a program to address the problem. On a quick pass through Lewis' reckoning of the some of the work done by discriminations of natural and non-natural properties there appeared to be enough affinity between, and overlap among mathematical and non-mathematical cases to give us hope. My first task in this paper was to illustrate, with reference to a representative instance, that appealing to metaphysical intuition (either directly or as coded into theoretical measures such as "simplicity" or "theoretical economy") to separate the cases where such choices of preferred "natural" categories are "merely pragmatic" and the cases where the choices are genuinely "objective" is unlikely to help us realize that hope, because of the fluidity and context-sensitivity of the relevant intuitions. We need some rough ground. The second goal was to point to one domain - the interaction between concept choice and successful prediction - where some such rough ground might be secured. A key point is that induction, as a pattern of reasoning under uncertainty, does not depend for its cogency on the predicted outcomes being contingent. Hence the link between assessments of the naturalness of properties and the correctness of predictions exhibited in "green" / "grue" cases carry over to mathematical cases. (The model of enumerative induction is just a handy orienting and simplifying device; the key foothold is the ubiquitous interaction in mathematical practice between choices of basic categories and the success of predictions (where the predictions may be clearly stated conjectures or less specific expectations of "fruitfulness")). Finally, I sketched the Riemann - Dedekind account of "inner characteristic properties" as a promising example of a method, and a methodology, incorporating this insight. This methodology has the additional attractive feature that in its details it resonates with the Port Royal principle, since identifiable mathematical cases drive it and give it substance. Of course, so far I've given only the roughest sketch of the Riemann - Dedekind account and the mathematics informing it, and I've given only unargued hints about its relationship to contemporary mathematical practice. In part this has been a function of space, but in part it is a necessity arising from the texts themselves. Riemann and Dedekind doled their methodological dicta out frugally. As mathematicians are prone to do, they let their mathematics do most of the talking, which leaves the philosopher/scribe a lot of detail to spell out. But this shouldn't be a surprize: Arnauld and Nicole warned us that this is what to expect. 
References

Armstrong, D. (1986) "In Defence of Structural Universals" Australasian Journal of Philosophy 64 pp.85 - 88

(1989) Universals: An Opinionated Introduction Boulder: Westview Press

Avigad, J. (2006) "Methodology and Metaphysics in the development of Dedekind's theory of Ideals" in José Ferreirós and Jeremy Gray, eds. The Architecture of Modern Mathematics Oxford: Oxford University Press pp. $159-86$

Benacerraf, Paul (1965) "What Numbers Could Not Be" The Philosophical Review 74 pp.47 - 73

Corfield, D. (2003) Towards a Philosophy of Real Mathematics Cambridge: Cambridge University Press

Cox, D. (1988) "Quadratic Reciprocity: Its Conjecture and Application" The American Mathematical Monthly 955 pp.442 - 448

(1989) Primes of the Form $x^{2}+n y^{2}$ New York: Wiley

Darrigol, O. (2005) Worlds of Flow: A History of Hydrodynamics from the Bernoullis to Prandtl Oxford: Oxford University Press

Dedekind R. (1854) "Über die Einfürung neuer Funktionen in der Mathematik" delivered as a Habilitation lecture June 30 1854, reprinted in Werke vol 3 pp. $428-438$

(1877/1996) Theory of Algebraic Integers J. Stillwell (trans. and ed.) Cambridge: Cambridge University Press

(1895) "Über die Begründung der Idealtheorie" Nachrichten der Königlichen Gesellschaft der Wissenschaften zu Göttingen pp. 106 - 113 ( = Werke II pp. $50-58)$

Dedekind R. and Weber, H. (1882) "Theorie der algebraischen Funktionen einer Veränderlichen" Journal für die Reine und Angewante Mathematik 92 
pp.181-290 ( = Dedekind Werke I pp. 248 - 349)

Edwards, H. (1980) "The Genesis of Ideal Theory" Archive for History of Exact Sciences 23 pp.321 - 378

(1983) "Euler and Quadratic Reciprocity" Mathematics Magazine 56 (5) pp. 285-291

Eisenbud, D. and Harris, J. (1992) Schemes: The Language of Modern Algebraic Geometry Boston: Wadsworth

Forrest, P. (1986) "Neither Magic nor Mereology: A Reply to Lewis" Australasian Journal of Philosophy 64 pp.89 - 91

Hilbert, D. (1897/1998) Theory of Algebraic Number Fields Ian T.Adamson (trans.) Berlin: Springer

Kleiman, S. and Laksov D. (1972) "Schubert calculus" American Mathematical Monthly 79 pp. 1061-1082

Lakatos, I. (1976) Proofs and Refutations, The Logic of Mathematical Discovery J. Whorrall and E. Zahar (eds.) Cambridge: Cambridge University Press

Lewis, D. (1983) "New Work for a Theory of Universals" Australasian Journal of Philosophy 61 pp.343 - 377 Reprinted in Lewis (1999)

(1984) "Putnam's Paradox" Australasian Journal of Philosophy 62 pp.221 Reprinted in Lewis (1999)

(1999) Papers on Metaphysics and Epistemology Cambridge: Cambridge University Press

(2001) "Redefining 'Intrinsic"' Philosophy and Phenomenological Research 63 pp.381 - 398

Mazur B. (1997) "Conjecture" Synthèse 111 pp.197-210

Riemann, B. (1851) "Grundlagen für eine allgemeine Theorie der Functionen einer veränderlichen complexen Grösse" Göttingen ( = Werke pp. 3 45)) 
(1857) "Beiträge zur Theorie der durch die Gauss'sche Reihe $F(\alpha, \beta, \gamma, x)$ darstellbaren Functionen" Abhandlungen der Königlichen Gesellschaft der Wissenschaften zu Göttingen $\mathbf{7}$ ( = Werke pp. 67 -83))

(1857a) "Abstract of [1857]" Göttinger Nachrichten 1 ( = Werke pp. 84 $85))$

(1857b) Theorie der Abel'schen Functionen (Journal für die reine und angewandte Mathematik $\mathbf{5 4}$ ( = Werke pp. 88 - 142))

Polyá, G. (1968) Mathematics and Plausible Reasoning (2nd ed.) in two volumes Princeton: Princeton University Press

Sandifer E. (2005) "How Euler Did It: Factors of Forms" MAA online column, December 2005 accessed from index: http://www.maa.org/news/howeulerdidit.html

Sider, T. (1996) "Naturalness and Arbitrariness" Philosophical Studies 81 pp.283-301

Stahl, H. (ed.) (1899) Elliptische Functionen: Vorlesungen von Bernhard Riemann Leipzig: Teubner

Tappenden, J. (2005) "The Caesar Problem in its Historical Context: Mathematical Background" Dialectica 59 fasc. 2 pp. 237 - 264

(200?) "Reflections on Mathematical Explanation: Why do Elliptic Functions have Two Periods?"

in preparation

Weatherson, B. (2006) "Natural Quantities" ms.

Weil A. (1984) Number Theory: An Approach through History; From Hammurapi to Legendre Boston: Birkhäuser

(1940/2005) "Letter of 1940 to Simone Weil" M. Krieger, (trans.) Notices of the American Mathematical Society vol 52 number 3 March 2005 pp.335 - 341 